\title{
BMJ Open Sustainability of community-based interventions for people affected by dementia: a protocol for the SCI-Dem realist review
}

\author{
Thomas Morton, ${ }^{\circ 1}$ Teresa Atkinson, ${ }^{1}$ Dawn Brooker, ${ }^{1}$ Geoffrey Wong, ${ }^{2}$ \\ Shirley Evans, ${ }^{1}$ Clive Kennard ${ }^{3}$
}

To cite: Morton T, Atkinson T, Brooker D, et al. Sustainability of community-based interventions for people affected by dementia: a protocol for the SCl-Dem realist review. BMJ Open 2019;9:e032109. doi:10.1136/ bmjopen-2019-032109

- Prepublication history and additional material for this paper are available online. To view these files, please visit the journal online (http://dx.doi. org/10.1136/bmjopen-2019032109).

Received 3 June 2019 Revised 11 June 2019 Accepted 12 June 2019

Check for updates

(c) Author(s) (or their employer(s)) 2019. Re-use permitted under CC BY-NC. No commercial re-use. See rights and permissions. Published by BMJ.

${ }^{1}$ Association for Dementia Studies, University of Worcester, Worcester, UK

${ }^{2}$ Nuffield Department of Primary Care Health Sciences, University of Oxford, Oxford, UK

${ }^{3}$ Library Services, School of Allied Health and Community, University of Worcester, Worcester, UK

Correspondence to

Dr Dawn Brooker;

d.brooker@worc.ac.uk

\section{ABSTRACT}

Introduction With numbers set to increase globally, finding ways to better support people with dementia and their families is a matter of growing concern. Community-based interventions can play a key role in supporting people with early to moderate stage dementia postdiagnosis, helping delay decline and hospitalisation. However, provision of such interventions is fragmented, with significant gaps and no reliable funding model, hence innovative groups and schemes catering for a genuine need can struggle long term and frequently fold.

Methods and analysis This realist review aims to expand our understanding of how best to implement and facilitate community-based interventions to run sustainably, focusing on contextually relevant explanations. We will gather and synthesise literature using a realist approach designed to accommodate and account for the complexity of 'real life' programmes, as implemented under different conditions in different settings, aiming to draw transferable conclusions about their sustainability that explain how and why context can influence outcomes. Our review will iteratively progress through five steps: (1) locate existing theories; (2) search for evidence (using Academic Search, AMED, CINAHL, EMBASE, MEDLINE, ProQuest, PsycINF0, PubMed, Scopus and Social Care Online, between May and September 2019); (3) article selection; (4) extracting and organising data; (5) synthesising the evidence and drawing conclusions. Data analysis will use a realist logic to explain what works, for whom, in what circumstances, in what respects, how and why. A stakeholder group will provide guidance and feedback throughout. Ethics and dissemination Ethical approval was not required. Recommendations drawn from results are likely to be of interest to a range of stakeholders including those commissioning, planning, running, supporting or attending such interventions, as well as policymakers, healthcare professionals and researchers. We will draw on the expertise of our stakeholder group regarding tailoring dissemination to each audience using a variety of materials, formats and channels.

\section{INTRODUCTION}

Postdiagnostic support for people living with dementia

More people are living with dementia worldwide, with numbers predicted to grow from
Strengths and limitations of this study

- This review will bring together learning from a wide range of place-based interventions aimed at supporting those affected by dementia living in the community postdiagnosis, to deepen our understanding how such interventions could be implemented more universally and consistently, long term.

- A realist approach is well suited to accommodate and account for the complexity of such 'real life' intervention programmes, as implemented under different conditions in different settings, to extract transferable conclusions.

- This will enable the development of recommendations and resources for those in a position to plan, commission, run or support such interventions or influence policy and infrastructure regarding themwhich is timely as there are currently gaps in such postdiagnostic support.

- This study is designed to gather evidence for how to successfully implement and sustain an intervention of this sort, not data on the effectiveness or otherwise of a particular intervention type.

- As this is a form of literature review, the richness of insight will depend on the depth and detail of information contained within the literature, which may be limited as this research question is not commonly the main focus of study in dementia care research.

46.8 million in 2015 to 131.5 million by $2050 .^{1}$ An increasing number of national strategies recommend diagnosis at earlier stages, as earlier diagnosis provides the opportunity for people and their families to make choices and lifestyle changes that will build resilience for the long term. $^{2}$ The evidence for the effectiveness of different types of postdiagnostic interventions is growing. ${ }^{34}$ However, in the UK, the availability and delivery of such early stage interventions is neither comprehensive nor uniform across regions and communities; instead, it is typically delivered sporadically and piecemeal by a mixed market of statutory, third sector, voluntary and 
community agencies with inconsistent funding, which can lead to fragmented provision with significant gaps.

Gaps in the care pathway for supporting those with early or moderate stage dementia can add to the confusion and fear that overwhelms families and people living with dementia when they need support. Budget constraints on statutory funding means the National Health Service (NHS) and local authorities are unable to prioritise people early on in their dementia who do not (as yet) have a significant level of need to reach the criteria for funding for care. Dementia advisers are now widely commissioned ${ }^{5}$ to provide sign-posting and some support, although formal evaluation suggests they need to be firmly embedded within local care pathways to work effectively. ${ }^{6}$ Family carers provide an enormous amount of care and support to people with dementia, yet the support they receive from the state varies markedly within the UK. Alzheimer and Memory Cafés are increasingly widespread, though for many a monthly meeting in a large group may not be enough to alleviate more significant needs to build long-term resilience.

\section{Benefits of meeting together in local communities}

Isolation and loneliness are major issues both for those living with dementia and family carers. ${ }^{78}$ Technology can help connect people but psychological theories suggest that the need for direct human contact is critical. ${ }^{9}$ There is evidence that regular social activity, where people are able to leave their homes and gather together in a communal setting on a frequent and ongoing basis, can be helpful both for people living with dementia and the people who care for them. ${ }^{10-15}$

The authors of this paper were engaged in the recent international MEETINGDEM project that investigated the implementation of the Dutch Meeting Centres Support Programme (MCSP) model in other European countries beyond the Netherlands. ${ }^{16}$ Meeting centres are venues that are based in a local community, facilitated by a small team of staff and volunteers trained in the ethos of person centred dementia care and the Adaptation-Coping Model. ${ }^{17}$ They were developed following a community needs assessment in the Netherlands 20 years ago and there are now $>140$ Dutch centres with a national infrastructure that local community groups can engage with to get help and support in bringing new centres on stream. Research has demonstrated that, compared with regular day-care, attending a meeting centre has a positive effect on mood, behaviour, self-esteem and delay of institutionalisation of the person with dementia ${ }^{10-12}$ and on sense of competence, burden, psychological and psychosomatic complaints of family caregivers. ${ }^{13}$ The MEETINGDEM project ran from 2014 to 2017 and successfully established and evaluated MCSPs in the UK, Italy and Poland. ${ }^{16}$ Those attending the meeting centres as part of the MEETINGDEM programmes reported significantly improved quality of life over a 6-month period compared with a control group receiving usual care. Higher attendance levels were associated with greater neuropsychiatric symptom reduction and increased feelings of support. ${ }^{14}$
Carers experienced less burden than those receiving usual care. $^{15}$

Meeting centres, however, are not the only such community-based intervention bringing people who are living with mild to moderate dementia together. The MEETINGDEM project brought the authors of this paper into contact with many other place-based day support initiatives that have a similar local ethos of bringing people together in a local community setting to provide postdiagnostic intervention. Many of these operate at a 'grass roots' level and in spite of the lack of any formal funding model for this kind of intervention. Some innovative enhanced day services also share elements in common with meeting centres. The similarities and differences between these well-established groups may point the way to how such interventions could be implemented more universally and consistently. It is timely to undertake a realist review investigating 'What makes a community intervention for people affected by dementia sustainable: in what circumstances and why?' The review aims to deepen our understanding of how best to implement and sustain such schemes for the benefit of those directly affected by dementia.

\section{METHODS}

\section{Aim}

To understand what makes a place-based community intervention for people affected by dementia sustainable long term (ie, for a period of 2 years or more).

\section{Objectives}

1. To conduct a realist review to understand what makes a place-based community intervention for people affected by dementia sustainable: in what circumstances and why?

2. To provide recommendations on what practices, resources and processes will best aid sustainability of such interventions to those in practice (whether in commissioning, planning or running), complete with accessible publications and online materials.

3. To provide recommendations to policymakers about what could be useful at both a regional and national level to aid the implementation and sustainability of such interventions.

\section{Review questions}

1. What are the mechanisms that determine whether people affected by dementia engage (or not) with community-based interventions?

2. What are the mechanisms that determine whether some interventions sustain over time and others do not?

3. What is the role of context in triggering the above mechanisms?

4. What context-sensitive resources, processes and behaviours are needed to ensure the sustainable implementation of a community-based intervention? 


\section{Patient and public involvement}

This study is focused on how to improve the long-term sustainability of existing community initiatives, a question raised as a challenging issue during the authors' work with those running and attending such initiatives during the MEETINGDEM project. ${ }^{16}$ Representatives of Innovations in Dementia CIC (Community Interest Company) and the DEEP (Dementia Engagement and Empowerment Project) were involved in the grant application to Alzheimer's Society at the start of this project. This study is primarily a literature review concerning community groups, so as such will not officially involve patient participants. However, in line with realist review guidelines (RAMESES: Realist And Meta-narrative Evidence Syntheses - Evolving Standards) ${ }^{18}$ a stakeholder group will be convened to advise and guide the project throughout, from the building of an initial theoretical model prior to our main search, to disseminating recommendations towards the end of the project. This group will include lay experts involved with community-based interventions in various capacities, whether commissioning, leading, running, supporting or attending. It is important to ensure that we hear from people directly affected by dementia, hence engaging with members of the DEEP network living with dementia will be part of this.

\section{Research plans}

Objective 1: to conduct a realist review

Our review will follow Pawson's five iterative stages ${ }^{19}$ as outlined in figure 1.

The realist review is an interpretive, theory-driven approach to synthesising evidence from a range of sources, including qualitative, quantitative and mixed-methods research. ${ }^{20}$ This approach is designed to accommodate and account for the complexity of 'real life' intervention programmes, as implemented under different conditions in different settings, aiming to explain how and why context can influence outcomes. ${ }^{21}$ Hence, it is well suited to extracting transferable conclusions from reviewing the functioning and success (or otherwise) of a range of community-based interventions for people affected by dementia, as these are likely to involve a high level of complexity and be responsive to contextual factors which are likely to vary considerably from intervention to intervention.

Explanation building in a realist review starts with the development and refinement of a realist programme theory of how community-based interventions for people affected by dementia are facilitated or impeded in their implementation and long-term sustainability. We will 'map' the factors involved and the steps needed for the long-term success of such an intervention, applying a realist logic of analysis-that is, how context may trigger certain mechanisms to lead to certain outcomes-to explain how intermediate outcomes for each step might be achieved. We define context as the conditions that trigger or modify the behaviour of mechanisms; ${ }^{22}$ Mechanisms are the usually hidden processes that generate outcomes, defined as 'underlying entities, processes, or structures which operate in particular contexts to generate outcomes of interest'. ${ }^{23}$

Our realist review protocol has been written by the project team and been registered with PROSPERO. ${ }^{24}$ The review is running for an 18-month period from December 2018.

\section{Step 1: locating existing theories}

This initial step is to identify and gather existing theories relating to how best to implement a community-based intervention for people affected by dementia sustainably, and what may impede this, to inform the review going forwards. This aims to include information on "what works for whom, in what circumstances, in what respects and how' ${ }^{21}$ This is based on the rationale that those who have designed and implemented the kind of interventions that are the subject of this review will have their own theories about how and why such interventions work and why certain components are required. ${ }^{19}$ For example, an early 2012 report on Debenham Project, ${ }^{25}$ a grass roots social activity and support initiative for people affected by dementia in and around the Suffolk village of Debenham-that has today been running for 10 yearsemphasised that factors such as a sense of ownership by a proactive community, as well as drive and vision from key scheme leaders, were essential to the project's growth and sustainability.

To locate such theories we have undertaken two parallel work streams, both to be carried out iteratively:

1. An exploratory, informal search of the literature aimed at quickly identifying the range of explanatory theories that may be relevant, while also helping us to become familiar with the range of initiatives of the kind that are the subject of this review that are in operation. This search is distinct from the more formal search outlined in step 2 as it is not systematic but rather uses informal search methods, such as citation tracking and snow-balling ${ }^{26}$ along with more structured but still informal scoping searches ${ }^{27}$ and the gathering of relevant publications and materials recommended by members of our stakeholder group (see below).

2. The convening of a stakeholder group, which will involve connecting with and consulting a range of key figures and lay experts, via focus group meetings, individual interviews, visiting community groups and email and telephone feedback on specific issues. In the first instance, we are asking two related questions: (A) what encourages or discourages people in making use of a community intervention? (barriers and facilitators to engagement); (B) what helps or hinders a community intervention in being sustainable long-term? (barriers and facilitators to sustainability). Once information from these questions has been collated and combined with what has been found by informal searching (see above), we will build an initial programme theory to test in the review. This will require iterative discussions 


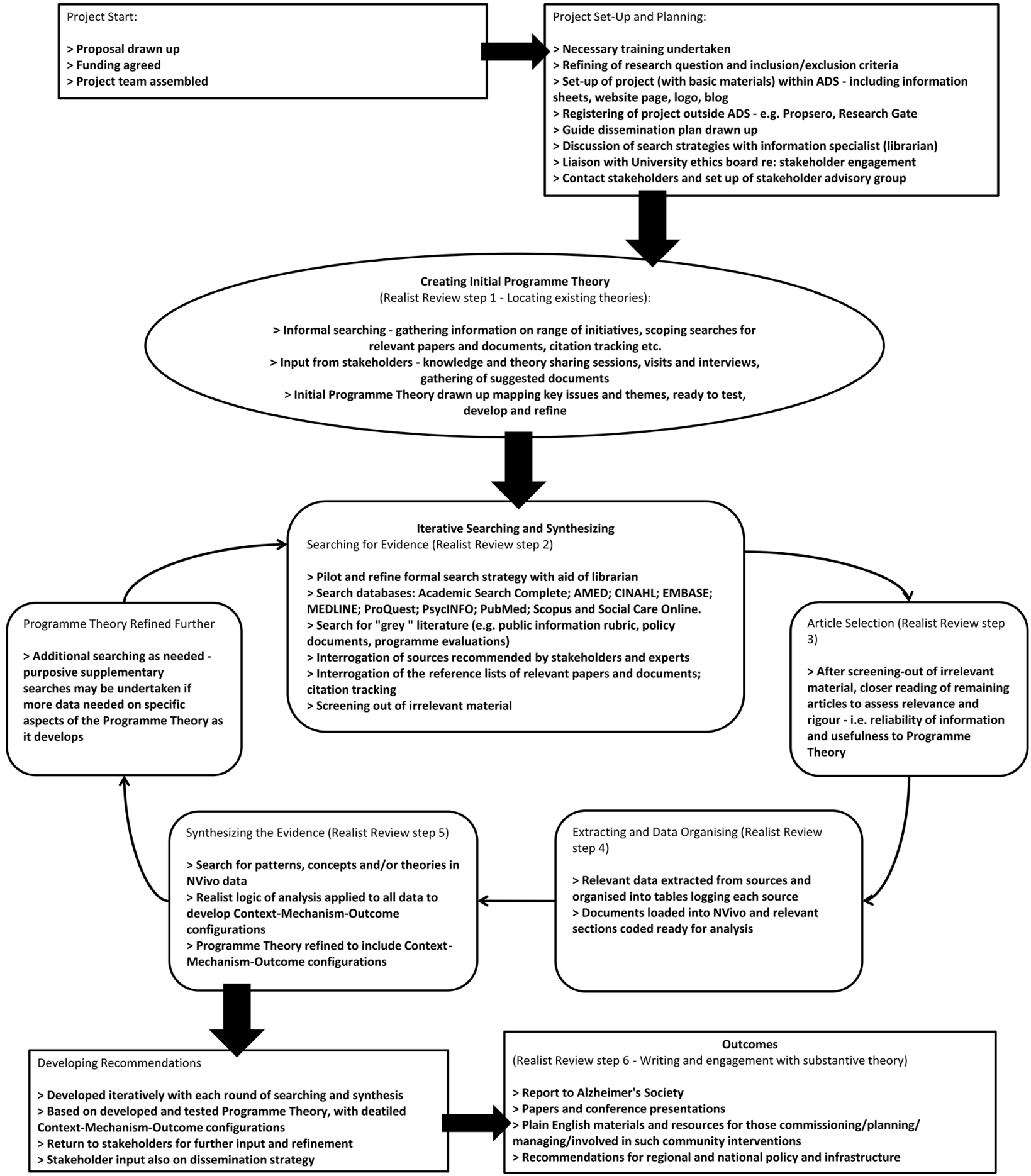

Figure $1 \mathrm{SCl}$-Dem project flow diagram.

within the project team to make sense of all the information. This initial programme theory will then be presented back to the stakeholder group to be refined based on their feedback. It will also inform our formal search strategy.

\section{Step 2: searching for evidence}

\section{Formal search}

Formal searching is being conducted to find a relevant 'body of literature' that might contain data to further develop and refine the programme theory. Multiple searches are anticipated. These are being designed, piloted and conducted by the research team with the guidance and assistance of an information specialist.

The following databases are being searched: Academic Search Complete; AMED; CINAHL; EMBASE; MEDLINE; ProQuest; PsycINFO; PubMed; Scopus and Social Care Online. Searches will take place between May and September 2019. Adaptations will be made for different databases if and as necessary. We may also search grey literature sources and other relevant bibliographic 
databases identified by our information specialist and will undertake forwards and backwards citation searches of relevant documents. Title, abstract and subject heading search terms directly related to our research question will be selected, tested and refined to find a balance between broad inclusivity and targeted relevance. Extra search terms, based on the input from stage 1 , will be used in further searches to narrow down the focus on specific issues (see online supplementary file 1).

In keeping with RAMESES guidelines, ${ }^{18}$ there are no restrictions on the types of study design eligible for inclusion. Documents such as editorials, opinion pieces, commentaries, process evaluations, qualitative research, programme manuals and systematic reviews may be included, if holding information relevant to developing a programme theory.

\section{Inclusion criteria}

Types of intervention for inclusion should:

1. Target people with mild to moderate dementia (whether exclusively or among others without dementia, but either way there is dementia-specific support).

2. Serve people living in the community, whether in their own homes or in extra-care housing.

3. Are voluntary attendance (ie, members have chosen to attend, not been told they must as part of treatment or respite care).

4. Are social and place-based (bringing people together physically) in a community setting (open to members of the public to attend).

5. Are designed as an intervention with meaningful activity aiming to improve quality of life for people with dementia and family carers, or to help them manage or lessen the challenging effects of dementia.

6. Meet at regular, prefixed times, at least weekly and for a substantial amount of time (ie, a morning or afternoon).

7. Meet continuously, on an ongoing basis, or aim to do so.

\section{Exclusion criteria}

Interventions will be excluded if they:

1. Are only for those with severe dementia.

2. Do not target, and have no plan to cater for, people with dementia.

3. Are only for care home residents, hospital patients or those in a closed institutional setting.

4. Are an online or at-a-distance networking scheme that does not involve meeting physically.

5. Only involve individual participants alone (eg, occupational therapy, counselling or medical).

6. Are only functional meetings solely for the purpose of administering medical treatment or carry out case management.

7. Are focused mainly on respite for carers or nursing care only (ie, not focused on social, meaningful and quality-of-life-raising activities for those attending).
8. Only take place monthly; or for a very short duration (eg, 1 hour); or intermittently with no specified or timetabled meetings.

9. Are fixed-term courses with a time/goal/session limit (eg, an 8-week course).

Screening will be undertaken first by title and abstract and then of the full text of documents. A $10 \%$ random subsample of the citations retrieved at each stage from searching will be reviewed independently by a second reviewer and any disagreements will be recorded and resolved by discussion. If disagreements still remain, then the matter will be resolved by discussion between the whole project team.

\section{Additional searching}

Searching is anticipated to be an iterative process, with extra targeted searches undertaken if more data are judged to be required to develop and test certain subsections of the programme theory. ${ }^{1820}$ We intend to carry out a series of targeted searches putting together key terms derived from the themes found during the input from stakeholder engagement and informal searching at stage 1. Areas that we believe may need additional searches include funding; implementation and engagement; network and marketing; and stigma and anxiety (see online supplementary file 1). Further areas may arise as the searching and analysis continues, as in a realist review this is an iterative process to be revised as necessary as the review progresses. ${ }^{18}$

If required, documents focused on interventions that only meet some, not all, of the inclusion criteria may be searched for to gather more information on specific hypothesised mechanisms, if there is reason to believe such mechanisms may function similarly or analogously in types of intervention that are closely related. ${ }^{19}$ For each additional search, the project team will meet to discuss and set inclusion and exclusion criteria. The screening processes will be as described above for the initial search.

\section{Step 3: article selection}

Article selection will be based on relevance and rigour: whether a document contains data that can contribute to developing or testing the programme theory; and whether those data were generated using credible and trustworthy methods. ${ }^{18} 20$ Data will be assessed on their own merits, not on that of the paper or study as a whole, as it is recognised that, for example, poorly designed or conducted research may still contain good quality 'nuggets' of information for a realist review. ${ }^{28}{ }^{29} \mathrm{~A}$ document that meets inclusion criteria may nevertheless not contain any relevant data for programme theory development and refinement.

The full text of all included papers will be read by one reviewer to decide if they contain data that could inform some aspect of the programme theory (relevance) while simultaneously making an assessment of rigour (the trustworthiness of data being used, where needed). ${ }^{20} 29$ A random sample of $10 \%$ of documents will be selected, 
assessed and discussed with a second reviewer to ensure that decisions to finally include a document based on relevance and rigour have been made consistently and to resolve any differences. The remaining $90 \%$ of decisions will be made by the first reviewer, though any ambiguous cases of relevance or rigour will be read by and discussed with the second reviewer and/or the wider project team as they arise. The relevance and rigour characteristics of each document will be logged on an EXCEL spreadsheet, along with information from step 4 .

\section{Step 4: extracting and organising data}

Data extraction and organisation will be by the first reviewer, again with a random $10 \%$ subsample reviewed independently by the second reviewer with any disagreements recorded and resolved by discussion. Any unresolved disagreements will be settled by discussion between the whole project team.

Full texts of included papers will be loaded into NVivo (qualitative data analysis software) to find and categorise (code) relevant sections of text that may relate to the developing programme theory, especially in regards to possible context-mechanisms-outcome configurations within it. Coding will be both inductive (codes created in response to data as it is found) and deductive (codes created in advance, informed by the initial programme theory). The characteristics of the documents, including the codes contained in each document, will be extracted separately into an EXCEL spreadsheet as previously mentioned in step 3. Included studies may be re-scrutinised iteratively as the programme theory develops.

\section{Step 5: synthesising the evidence and drawing conclusions}

Realist logic will be used to make sense of the initial programme theory and synthesise data in relation to it. ${ }^{18}$ Interpretive cross-case comparison will be used to understand and explain how and why observed outcomes have occurred-for example, by comparing interventions which have sustained robustly with a high level of engagement with those which have had less sustained success in implementation over time, to understand how context may have influenced the sustainability of a project or group. Likely context-mechanism-outcome configurations will be identified and mapped based on the data, from which conclusions can be drawn about their role within the overall programme theory.

While the processes above have been set out above in a linear way, in a realist review they can be undertaken iteratively and overlap to a significant degree. The purpose of analysis and synthesis is to understand how mechanisms behave under the different contexts described within the documents included in the review. Figure 1 illustrates the processes discussed and where there may be iterative elements.

\section{Objectives 2 and 3: to provide recommendations}

Our refined programme theory will inform the development of publications and materials to make recommendations, to both policymakers and those in practice, on how to best aid the implementation and sustainability of community-based interventions for people affected by dementia. Further details are provided in the Dissemination plans and Project outputs sections.

\section{DISSEMINATION PLANS}

The results of our review are likely to be of interest to a range of stakeholders including

1. Those planning to run, or currently running, a community-based intervention.

2. Those attending, or involved with supporting, a community-based intervention.

3. Those responsible for commissioning such services/ support/interventions.

4. Those responsible for setting policy regarding dementia-related services/support/interventions.

5. Fellow researchers in the dementia and health and social care field.

Different strategies are likely to be needed for such diverse audiences and our approach will be integrative, aiming to recognise and value the different forms of knowledge needed to inform complex decision-making. ${ }^{30}$ We will draw on the advice and expertise of our stakeholder group to help clarify who is best placed to contact regarding dissemination for each audience and what kind of materials and information may be most relevant to them, as well as taking advice directly from such suggested contacts on preferred materials, formats and channels. For example, a full end-of-study report will be produced for the Alzheimer's Society, academic papers produced for submission to peer-reviewed journals and professional conference presentations planned and delivered. In addition, for a non-academic and general public audience, materials in various formats (including online materials) will be developed with 'plain English' summaries of the review findings and recommendations. Throughout the review process an online project 'blog' (https://scidemreview.wordpress.com/) will be kept and publicised via social media and in newsletter articles.

\section{PROJECT OUTPUTS}

This review aims to produce the following as its main outcomes:

1. A programme theory for effective implementation of sustainable, place-based community interventions, including mapping of frequently occurring features that may inhibit or promote the success of a scheme, including (1) a detailed outline of contextually informed barriers and facilitators to engagement with social and community schemes designed to aid mood, cognitive functioning, mobility and physical health, and delay decline and hospitalisation in those with mild to moderate dementia; (2) a detailed outline of contextually informed barriers and facilitators to the continued provision of social and community schemes designed 
to aid mood, cognitive functioning, mobility and physical health, and delay decline and hospitalisation in those with mild to moderate dementia.

2. Recommendations to those commissioning, planning and running place-based community interventions.

3. Recommendations for national and/or regional infrastructure to support local community support.

4. The development of useful and accessible materials and resources to communicate the above recommendations to target audiences.

5. A report to Alzheimer's Society (project funders), academic papers and conference dissemination.

As additional outcomes, this review aims to:

1. Enable further research in the form of a realist evaluation building on what is found/the programme theory developed in this review.

2. Encourage and support the implementation and success, and/or improvement and continuation, of high quality initiatives in place-based community provision for those affected by dementia and their carers.

\section{DISCUSSION}

Dementia is being diagnosed earlier and the number of people living with dementia is expected to rise significantly in the coming decades. This is a global trend. ${ }^{1}$ However, strategies to support any but those with the most acute needs tend to be underdeveloped and inconsistently applied in the UK at present. While there is a demand for early stage postdiagnostic support and a grass roots drive to provide it, reliable funding and recognition within the health and social care infrastructure is rare for these kind of interventions. As a result such initiatives have a tendency to collapse and disappear within a couple of years of starting despite much expense, hard work and innovation in setting up, a real need in communities and appreciation from those who use such services.

The literature so far has tended to focus only on whether different kinds of psychosocial interventions work or not. There is growing evidence that interventions involving regular social meetings and activities are beneficial for people living with dementia in the early to moderate stages. ${ }^{10-15}$ However, the factors involved are likely to be extremely complex and any beneficial effects are useless if the delivery of such interventions cannot be sustained. This realist review seeks to look at the wider contextual drivers for whether a community-based intervention can be implemented successfully and kept going in the long term. An increased understanding of what facilitates or impedes this will be used to develop recommendations and resources for those in a position to plan, commission, run or support such interventions, or influence policy and infrastructure regarding them. No prior realist review has been undertaken on this.

\section{Importance of the research}

With the number of people in society living with dementia set to increase, finding ways in which we can better support both people with dementia and their families and carers is a growing concern. There is evidence that community-based groups and initiatives can play a key role in everything from combating isolation and maintaining positive self-image to delaying decline and hospitalisation. This realist review will expand our understanding of how best to facilitate such interventions so that they can sustain long term by focusing on contextually relevant explanations. This in turn will develop outputs to inform the planning and implementation of future interventions, and the policy surrounding this.

\section{Twitter Follow Thomas Morton @ThomasMortonADS}

Acknowledgements This project was funded by the Alzheimer's Society. The authors would like to thank all those who shared their invaluable experience and contributed to advising and guiding this project as a stakeholder consultant.

Contributors DB, TA and SE conceptualised the study with input from GW, TM and CK. TM wrote the first draft of this manuscript. GW, DB and TA critically contributed to and refined this manuscript. All authors have read and approved the final manuscript.

Funding The Alzheimer's Society (Grant No: 402, AS-PG-17b-023).

Disclaimer The views and opinions expressed therein are those of the authors and do not necessarily reflect those of the Alzheimer's Society.

Competing interests GW is Joint Deputy Chair of the National Institute for Health Research Health Technology Assessment Programme Prioritisation Committee Panel A (Out of hospital).

Patient consent for publication Not required.

Ethics approval This project has been reviewed by the relevant University of Worcester Ethics Committee.

Provenance and peer review Not commissioned; peer reviewed for ethical and funding approval prior to submission.

Open access This is an open access article distributed in accordance with the Creative Commons Attribution Non Commercial (CC BY-NC 4.0) license, which permits others to distribute, remix, adapt, build upon this work non-commercially, and license their derivative works on different terms, provided the original work is properly cited, appropriate credit is given, any changes made indicated, and the use is non-commercial. See: http://creativecommons.org/licenses/by-nc/4.0/.

\section{REFERENCES}

1. Alzheimer's Disease International. World Alzheimer Report 2015. The global impact of dementia: an analysis of prevalence, incidence, cost and trends. London: Alzheimer's Disease International, 2015.

2. Brooker D, La Fontaine J, Evans S, et al. Public health guidance to facilitate timely diagnosis of dementia: ALzheimer's COoperative Valuation in Europe recommendations. Int J Geriatr Psychiatry 2014;29:682-93.

3. MODEM Project. The Dementia Evidence Toolkit. $2016 \mathrm{http} / / /$ toolkit. modem-dementia.org.uk/.

4. British Psychological Society. A guide to psychosocial interventions in early stages of dementia; faculty of psychology and older people. Leicester: British Psychological Society, 2014.

5. Mori I. Survey of provision of dementia adviser services. London: Ipsos Mori, 2016.

6. Clarke CL, Keyes SE, Wilkinson $\mathrm{H}$, et al. HEALTHBRIDGE; The National Evaluation of Peer Support Networks and Dementia Advisers in implementation of the National Dementia Strategy for England [Ref: 025/0058] Department of Health Policy Research Programme Project. London: Department of Health, 2013.

7. Alzheimer's Society. Dementia 2013: the hidden voice of loneliness. London: Alzheimer's Society, 2013.

8. Alzheimer's Society. Dementia 2014: opportunity for change. London: Alzheimer's Society, 2014.

9. Pinker S. The village effect: why face-to-face contact matters. London: Atlantic Books, 2015.

10. Dröes RM, van der Roest HG, van Mierlo L, et al. Memory problems in dementia: adaptation and coping strategies and psychosocial treatments. Expert Rev Neurother 2011;11:1769-82. 
11. Dröes RM, Breebaart E, Ettema TP, et al. Effect of integrated family support versus day care only on behavior and mood of patients with dementia. Int Psychogeriatr 2000;12:99-115.

12. Dröes RM, Meiland F, Schmitz M, et al. Effect of combined support for people with dementia and carers versus regular day care on behaviour and mood of persons with dementia: results from a multi-centre implementation study. Int J Geriatr Psychiatry 2004;19:673-84.

13. Dröes RM, Meiland FJ, Schmitz MJ, et al. Effect of the meeting centres support program on informal carers of people with dementia: results from a multi-centre study. Aging Ment Health 2006;10:112-24.

14. Brooker D, Evans S, Evans S, et al. Evaluation of the implementation of the Meeting Centres Support Program in Italy, Poland, and the UK; exploration of the effects on people with dementia. Int J Geriatr Psychiatry 2018;33:883-92.

15. Evans S, Evans S, Brooker D, et al. The impact of the implementation of the Dutch combined Meeting Centres Support Programme for family caregivers of people with dementia in Italy, Poland and UK. Aging Ment Health 2018:1-11.

16. Mangiaracina F, Chattat R, Farina E, et al. Not re-inventing the wheel: the adaptive implementation of the meeting centres support programme in four European countries. Aging Ment Health 2017;21:40-8.

17. Brooker D, Dröes R-M, Evans S. Framing outcomes of postdiagnostic psychosocial interventions in dementia: the AdaptationCoping Model and adjusting to change. Working with Older People 2017;21:13-21.

18. Wong G, Greenhalgh T, Westhorp G, et al. Development of methodological guidance, publication standards and training materials for realist and meta-narrative reviews: the RAMESES (Realist And Meta-narrative Evidence Syntheses - Evolving Standards) project. Health Serv Deliv Res 2014;2:1-252.
19. Pawson R, Greenhalgh T, Harvey G, et al. Realist synthesis - an introduction. ESRC Working paper series. London: ESRC, 2004.

20. Pawson R. Evidence-based policy: a realist perspective. London: Sage Publications, 2006

21. Pawson R, Greenhalgh T, Harvey G, et al. Realist review - a new method of systematic review designed for complex policy interventions. J Health Serv Res Policy 2005;10:21-34.

22. Jagosh J, Macaulay AC, Pluye P, et al. Uncovering the benefits of participatory research: implications of a realist review for health research and practice. Milbank Q 2012;90:311-46.

23. Astbury B, Leeuw FL. Unpacking black boxes: mechanisms and theory building in evaluation. Am J Eval 2010;31:363-81.

24. Booth $A$, Clarke M, Ghersi D, et al. An international registry of systematic-review protocols. Lancet 2011;377:108-9.

25. Lockwood S. The Debenham project: a case study of a unique community-led and owned project dedicated to the support of carers of those that have symptoms of dementia and those they care for. Harrogate: Community Catalysts CIC, 2012.

26. Greenhalgh T, Peacock R. Effectiveness and efficiency of search methods in systematic reviews of complex evidence: audit of primary sources. BMJ 2005;331:1064-5.

27. Booth A, Harris J, Croot E, et al. Towards a methodology for cluster searching to provide conceptual and contextual "richness" for systematic reviews of complex interventions: case study (CLUSTER). BMC Med Res Methodol 2013;13:118.

28. Pawson R. Digging for nuggets: how 'bad' research can yield 'good' evidence. Int J Soc Res Methodol 2006;9:127-42.

29. Wong G. Data gathering in realist reviews: Looking for needles in haystacks. In: Emmel N, Greenhalgh J, Manzano A, eds. Doing Realist Research. London: Sage Publications, 2018:131-45.

30. Bowen S, Graham I. Integrated knowledge translation. In: Straus S, Tetroe J, Graham I, eds. Knowledge translation in health care: moving from evidence to practice. Oxford: Wiley Blackwell, 2013:14-23. 